Voix et Images

voixetimages

\title{
Tardivel et le roman chrétien de combat
}

\section{Bernard Andrès}

Volume 2, numéro 1, septembre 1976

Fernand Leduc

URI : https://id.erudit.org/iderudit/200024ar

DOI : https://doi.org/10.7202/200024ar

Aller au sommaire du numéro

\section{Éditeur(s)}

Les Presses de l'Université du Québec

\section{ISSN}

0318-9201 (imprimé)

1705-933X (numérique)

Découvrir la revue

\section{Citer cet article}

Andrès, B. (1976). Tardivel et le roman chrétien de combat. Voix et Images, 2(1), 99-109. https://doi.org/10.7202/200024ar d'utilisation que vous pouvez consulter en ligne.

https://apropos.erudit.org/fr/usagers/politique-dutilisation/ 


\section{Tardivel et le roman chrétien de combat}

Pour la Patrie. Roman du XXe siècle, est la marque d'une double outrance à la fin du XIXe siècle québécois. Outrance idéologique du texte ultramontain qui pousse jusqu'à l'excès les théories intégristes et l'appel à une théocratie nationaliste. Outrance littéraire du "roman chrétien de combat " (Tardivel dixit), sorte de thèse romanesque historico-sentimentale, d'aventure et d'anticipation. Le plus intéressant dans le roman à thèse, c'est moins l'idéologie véhiculée (la plupart du temps, elle se trouve formulée de façon maladroite et perd considérablement à ne pas l'être sous une forme plus adéquate: essai, pamphlet, etc.), que la façon dont celle-ci articule le roman, définit un certain nombre de contraintes discursives et produit en fin de compte une forme spécifique. La critique peut en rendre compte en montrant comment le texte produit respecte ou non, rejette ou intègre les lois du roman 1 .

L'intégration de toutes les composantes du genre hybride évoqué plus haut pose au critique le problème des rapports entre idéologie et forme romanesque, dans une perspective socio-historique du phénomène littéraire. Comment peut-on écrire Pour la Patrie en 1895? Et pourquoi? (Hasard ou nécessité de cette œuvre dans la production littéraire/sociale de l'époque?) Les deux questions se recoupant en un sens, ces quelques pages s'arrêteront surtout à la première interrogation. Elles tenteront de montrer comment des éléments romanesques aussi disparates peuvent aisément composer (dans) un texte à prétention narrative. Peut-être pourrons-nous en traitant de ce problème, aborder la seconde quéstion et, sans la régler, y apporter tout au moins des éléments de réponse. Pour la méthode, on établira d'abord l'encodage du texte en fonction de l'Avantpropos de l'auteur, puis l'on comparera le fonctionnement du discours romanesque à cet encodage.

\section{LE PACTE ROMANESQUE DE TARDIVEL}

Tardivel embarrasse la critique universitaire. Dans son patient effort pour diviser la production romanesque "canadienne-française" en trois 
types de romans «qui naissent et se développent parallèlement: le roman d'aventures, le roman historique et le roman de la terre", Paul Wyczynski s'interroge: "que dire de Jules-Paul Tardivel qui, dans Pour la Patrie (1895), donne une vision fausse de la réalité future, dans le sens d'un nationalisme outré et démodé ${ }^{2}$ ? On cherche en vain les critères de cette "fausseté", même si cette vision n'est pas si fausse en un sens. Mais peu importe. Ce premier roman séparatiste québécois nous intéresse moins par sa conformité au "réel " (du critique), que par l'écart qu'il accuse:

- au niveau des structures romanesques de l'époque, et

— au niveau d'une outrance idéologique voisine de l'écriture pamphlétaire.

Pour situer synchroniquement le roman, on utilisera les précieuses indications de John Hare ${ }^{3}$, ainsi que l'Avant-propos de l'auteur.

Si, comme le note Philippe Lejeune, "une poétique historique se devrait [...] d'étudier l'évolution du système des contrats de lecture et de leur fonction intégrante ${ }^{4}$, il convient de définir le pacte conclu entre l'auteur de notre Avant-propos et son allocutaire ainsi mis en garde: "Qu'on ne cherche donc pas dans ces pages le fini exquis des détails qui constitue le charme de beaucoup de romans. " A s'en tenir au contenu manifeste de ces lignes, on ne peut s'attendre à grand chose au niveau narratif: “Je n'ai pas la prétention d'offrir au public une œuvre littéraire délicatement ciselée, ni une étude de mœurs patiemment fouillée; mais une simple ébauche où, à défaut de gracieux développements, j'ai tâché de mettre quelques idées suggestives que l'imagination du lecteur devra compléter. "

Faut-il se méfier de la fausse modestie propre à l'Avant-propos? John Hare insiste avec raison sur le caractère très élaboré de ce roman à thèse. En fait, seuls les «grands développements" sont rejetés au profit de la clarté et de la concision: en vingt ans de journalisme polémique, explique Tardivel, “j'ai contracté l'habitude de condenser ma pensée, de l'exprimer en aussi peu de mots que possible, de m'en tenir aux grandes lignes, aux points principaux". On verra effectivement qu'il s'agira d'une intrigue simple, voire unique. Pour ce qui est du style, l'auteur rejetterait toute "fleur", tout "ornement" rhétorique. Qu'adviendra-t-il de ce projet déjà battu en brèche dans l'Avant-propos par les chapelets métaphoriques sur «la fleur d'espérance» plantée «dans le cœur de tout Canadien français patriote" et sur ala vertu chrétienne qui travaille autant qu'elle prie; qui plante, qui arrose et qui attend de Dieu la moisson 5 ?

Une première opposition se fait jour entre les structures narratives et les tournures stylistiques du texte: simplicité vs recherche. Cette opposition reflète en fait une contradiction fondamentalement idéologique; tout en rejetant le roman: "Le roman, surtout le roman moderne, et plus particulièrement encore le roman français me paraît être une arme forgée par Satan lui-même [...] " Tardivel reconnaît en écrire un! Contradiction aussitôt rejetée "pour la raison qu'il est permis de s'emparer des machines de guerre de l'ennemi et de les faire servir à battre en brèche les remparts 
qu'on assiège". Sans s'arrêter sur la valeur de l'argumentation, on insistera ici sur ce réflexe du rejet des valeurs étrangères à l'auteur, ou plutôt de la substitution de celles-ci par d'autres plus personnelles.

La tournure substitutive de ces formules:

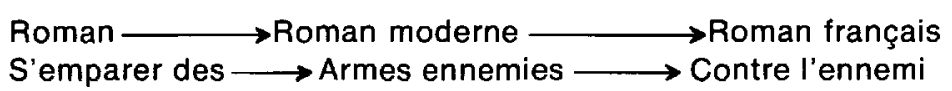

définit un principe d'escamotage, de permutation, encodé dès l'Avantpropos et dont le roman se ressentira. Ainsi, parallèlement à la vieille dichotomie “Bien-Mal», réapparaît celle du fond et de la forme. Les fins du roman «infâme» sont rejetées; mais ses moyens - structures formelles - se trouvent intégrées dans un nouveau système narratif: le “ROMAN CHRÉTIEN DE COMBAT". II s'agit d'un nouveau type de récit “qui fortifie la volonté, qui élève et assainit le cœur, qui fait aimer davantage la vertu et haïr le vice, qui inspire de nobles sentiments, qui est en un mot, la contre-partie du roman infâme ${ }^{6}$ ".

L'objectif d'une telle entreprise d'édification: “[...] l'aspiration vers l'établissement, sur les bords du Saint-Laurent, d'une Nouvelle-France dont la mission sera de continuer sur cette terre d'Amérique l'œuvre de civilisation chrétienne que la vieille France a poursuivie avec tant de gloire pendant de si longs siècles ${ }^{7}$. M $^{9 r}$ Laflèche et Philippe Masson ne sont pas loin. Tout l'esprit missionnaire et la tradition providentialiste de "notre mission comme peuple» réapparaissent, repris et prolongés par Tardivel. Car John Hare note bien qu'avant ce dernier, les penseurs nationalistes "s'arrêtent à l'exposition de la mission, sans tirer la conclusion politique qui s'impose. S'ils reconnaissent l'existence d'une nation canadienne-française, ils ne voient pas la nécessité d'une expression politique de cette nation. Tardivel demeure ainsi le premier à relier directement la mission providentielle des Canadiens-français à la création d'un état catholique au Québec ${ }^{8}$. Ainsi donc, Tardivel prolonge et réalise la pensée ultramontaine québécoise dans un ouvrage d'anticipation à court terme: "à l'encontre des romans utopistes, Tardivel ne décrit pas la société parfaite mais plutôt les étapes vers son établissement. En situant son roman en 1945, cinquante ans après la date de publication, il se donne rendezvous avec une date qui n'est pas dans un lointain fantastique. Selon Raymond Ruyer, «l'anticipation à terme est la forme la plus «scientifique» de l'utopie. L'expérience mentale n'y prétend que prolonger l'expérience historique elle-même ${ }^{9}$.

Tardivel choisit-il le demi-siècle «pour marquer sa foi dans l'imminence des événements qu'il décrit», comme le pense John Hare? La réponse est encore une fois dans l'Avant-propos. Le choix du rapport temps du discours est intimement lié à un impératif idéologique: entre la temps du récit

hâte du matérialisme progressiste et l'inertie d'un providentialisme fataliste, se place, pour Tardivel, «la vertu chrétienne qui travaille autant qu'elle prie»; car "l'homme peut et doit travailler à empêcher que cette heure 
providentielle ne soit retardée ". Situation des plus inconfortables puisqu'en d'autres termes, il faut agir sans agir (seule agit en droit la Providence). Tardivel ne peut se contenter de la Vérité (journal où il analyse et dénonce le réel). Seule la fiction lui permet de trans-former ce réel, de le transposer d'une temporalité à l'autre, dans un avenir purement fictif puisqu'équivalant à un retour au passé, à ce “Moyen-Âge idéalisé " dont parle Ruyer à propos des écrivains catholiques. Selon Mgr Fèvre, biographe de l'auteur, "à l'instar de Charlemagne, [Tardivel] rêvait d'une chaîne de monastères entourés de colons “des Laurentides à Vancouver ${ }^{10}$ ".

Ressortent de cet Avant-propos un certain nombre de constantes qu'il convient à présent de synthétiser.

Le pacte romanesque de Tardivel s'apparente à son contrat de polémiste ( condenser ma pensée, [...] m'en tenir aux grandes lignes, aux points principaux»). L'idée centrale (fixe?) consiste dans l'opposition des forces du Bien et de celles du Mal, les premières tentant de se substituer aux autres en les intégrant à leur système dans/par la fiction romanesque ${ }^{11}$. Nous nous interrogerons désormais sur la fonction structurale des éléments «substitution - intégration", si fortement encodés dans l'Avantpropos, afin de voir si le pacte romanesque défini plus haut est respecté dans l'ensemble du texte. Un parallélisme pourrait alors s'établir entre l'idéologie intégriste de l'auteur et le principe intégrateur du croman chrétien de combat ", sorte de permutation des extrêmes. Cette dynamique déterminerait le fonctionnement textuel, depuis l'ouverture sur les premières unités actantielles (chapitres 1 et 2), jusqu'à une clôture marquant l'épuisement des possibilités combinatoires de ces unités (chapitre 33 et Épilogue).

II ne saurait être question de rendre compte ici de la structure romanesque dans son ensemble. Le principe "PERMUTATION-INTÉGRATION" fonctionne tout aussi bien au niveau du tressage de la narration (jeu complexe et subtil des amorces, annonces, leurres et faux-leurres; $c f$. Barthes), que des fonctions du narrateur (fonction de régie vs fonction idéologique; cf. Genette). Je ne ferai donc qu'illustrer ce principe par quelques remarques sur deux autres éléments constitutifs du texte.

\section{PERMUTATION - INTÉGRATION DES TYPES ROMANESQUES}

Si le roman de Tardivel peut dérouter une critique taxonomique, c'est qu'il présente un cas de transgression typologique. En concevant avec France Vernier les formes littéraires comme "des formations de compromis signifiantes résultant de forces antagonistes", on pourrait mettre en relation le cas de Pour la Patrie avec la manière de compromis idéologique dont témoignent les prises de position politiques de Tardivel. Là n'est pas notre propos; nous nous contenterons de remarquer que les systèmes romanesques évoluent énormément au XIX siècle. Au Québec principalement, où le genre remonte tout juste à «l'année fatale $1837^{12}$ ", 
Pour la Patrie offre un cas de transgression du genre, au sens que Ducrot et Todorov donnent à l'expression ${ }^{13}$.

Rappelons brièvement les genres auxquels se rattache notre texte, en notant comment, tout en s'intégrant à la structure d'ensemble, ils se substituent indéfiniment les uns aux autres selon la perspective narrative ou le mode de décodage adoptés. On recourt ici à la typologie du roman traditionnel dont s'inspirent Wyczynski et Hayne dans le Roman canadienfrançais ${ }^{14}$.

\section{Roman d'intrigue et roman historique}

Pour la Patrie allie aux péripéties du roman d'intrigue l'arrière-plan événementiel du roman historique, les deux genres s'escamotant tour à tour suivant le principe de permutation évoqué plus haut. La gravité de l'enjeu frisant l'épique («le salut d'une race en Amérique du Nord»), le second genre l'emporte le plus souvent sur le premier. Qu'on en juge par l'exposé de la situation; l'Angleterre rompant les ponts avec le Canada, celui-ci doit choisir entre trois voies: le statu quo, l'union législative ou la séparation. Dans le premier cas, la confédération: transition en douceur avec les modérés du parti conservateur, la corruption, le favoritisme, etc. L'union législative, c'est le radicalisme à outrance des francs-maçons, "ennemis déclarés de l'Église et de l'élément canadien-français", avec à leur tête, le «suppôt de Satan" Montarval, le journaliste Ducoudray et - comble de la perfidie! - le premier ministre conservateur Sir Henry. Enfin, les séparatistes, avec Lamirande et Leverdier, prônent pour le $\mathrm{Ca}$ nada français "un État séparé et indépendant: la Nouvelle-France», théocratie à la Garcia Moreno ${ }^{15}$.

Si l'on accepte les abréviations suivantes:

$L M=$ Lamirance $; L V=$ Leverdier; SS = Saint-Simon; DC = Ducoudray; $M T=$ Montarval $; \mathrm{SH}=$ Sir Henry $;(D)=$ Dieu et $(S)=$ Satan ;

le schéma actantiel se présente ainsi :

HÉROS ADJUVANTS TRAÎTRES OPPOSANTS

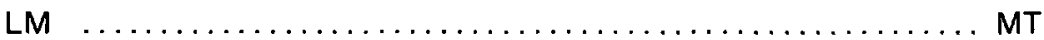

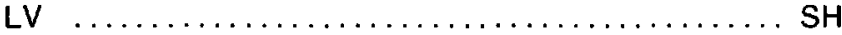

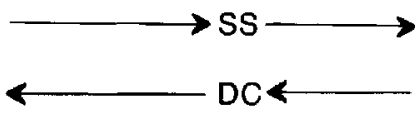

(D) 
A tout instant la lecture bascule d'un type de décodage romanesque à l'autre. Ainsi lors des moments de crise où le héros songe à ses malheurs, ou juste avant un acte perfide de l'ennemi ${ }^{16}$. L'élan narratif est alors brisé: on passe au registre de la fonction idéologique du locuteur... pour bientôt retomber dans les méandres d'une intrigue politique alternant à l'occasion avec quelque histoire de cœur.

\section{Le roman sentimental}

Apparemment relégué à l'arrière-plan, il n'en existe pas moins, intégré à l'histoire affective du héros. Pour reprendre les abréviations: LM épouse Marguerite, scur adoptive d'Hélène qui aimera toute sa vie en secret LM (elles sont toutes deux scurs de l'adjuvant LV). Cette histoire de cœur apparaît aux chapitres 2 et 3 pour disparaître à l'Épilogue, enchâssant (ou enchâssée dans) les séquences intermédiaires. Autre exemple d'intégration parfaite: aucune gratuité dans cette séquence narrative qui permet d'inscrire dans la série des épreuves subies par LM, l'empoisonnement de sa femme (tout en confirmant le principe de permutation $M G=$ Marguerite et $\mathrm{HL}=$ Hélène $-:$ :

\section{Le roman merveilleux et le merveilleux instrumental}

Je regroupe ici avec Todorov deux types romanesques assez proches dans la mesure où ils font appel à des éléments surnaturels ou inhabituels, mais acceptés comme tels par le personnage et/ou le lecteur ${ }^{17}$. On prie beaucoup dans Pour la Patrie, aussi bien Dieu que le Diable. Prières, invocations, apparitions et miracles se suivent et s'annulent mutuellement jusqu'à la victoire finale du Bien:

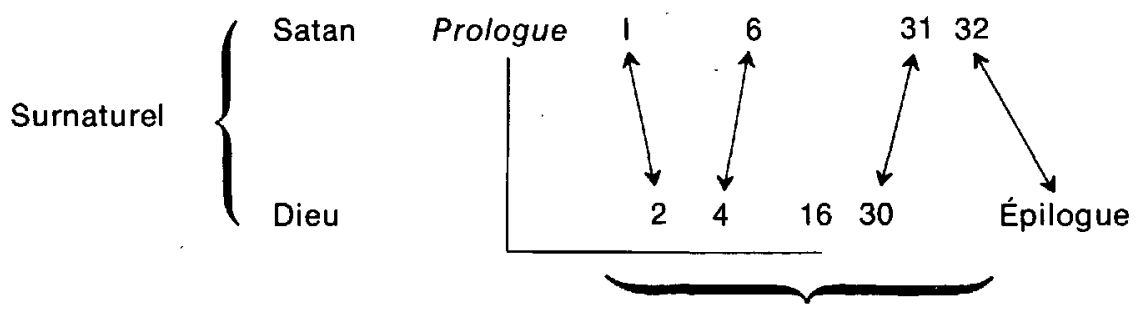

Chapitres

Permutations sérielles analogues à celles relevant du «merveilleux instrumental ": "Apparaissent ici de petits gadgets, des perfectionnements techniques irréalisables à l'époque décrite, mais après tout parfaitement possibles ${ }^{18}$." L'intégration diégétique de ces éléments est assez admirable: 
la «plume télégraphique» permettant aux francs-maçons de communiquer leurs sombres desseins, verra sa fonction inversée: le dernier “télex" de Montarval, mal interprété par Duthier, coûtera la vie à ce dernier (chapitre $18 \leftrightarrow$ chapitre 26). De façon générale, tout substitut du progrès matériel se retourne contre les matérialistes, témoin la mort de Duthier dans cet accident ferroviaire ponctué par: "La pauvre humanité venait d'offrir un nouvel holocauste au dieu Progrès ${ }^{19}$. »

Nous touchons là au jeu des permutations actantielles, sur lesquelles nous choisirons de clôturer ces remarques.

\section{PERMUTATIONS ACTANTIELLES DES PERSONNAGES-TRANSFUGES}

La distribution des personnages dans cette thèse romanesque obéit à la vision manichéenne évoquée plus haut. II s'agit donc d'une structure actantielle résolument binaire. Caractères tranchés d'un bout à l'autre du roman, avec plongées omniscientes dans la «psychologie " des héros. Deux pôles se détachent: Lamirande, auréolé de bonté et de persévérance et Montarval, sombre étranger échoué dans un roman nationaliste, suppôt de Satan par qui «la France mondaine, sceptique, railleuse, impie et athée [...], ennemie de Dieu et de son Église a fait irruption au Canada»! Gravitent autour d'eux la nébuleuse des opposants et adjuvants parmi lesquels nous choisirons les plus intéressants (textuellement parlant): les personnages-transfuges. Non seulement mieux analysés (les fluctuations narratives dépendant de leurs états d'âme), ce sont eux qui défendent et illustrent le mieux le bien-fondé de la juste cause... et de notre principe d'intégration-permutation. II s'agit de Saint-Simon, directeur du Progrès catholique, de Ducoudray, de la Libre Pensée, et de Vaughan, député anglais. Développons le schéma actantiel esquissé plus haut. Si l'on appelle pour les besoins de la schématisation, du côté des «forces du Bien»:

$B-B^{\prime}=$ le couple des héros Lamirande - Leverdier, avec leurs adjuvants:

$\mathrm{B}_{1}=$ Saint-Simon; $\mathrm{B}_{2}=$ Marguerite et $\mathrm{B}_{3}=$ Marie (fille de LM);

et du côté des «forces du Mal»:

$M-M^{\prime}=$ le couple Montarval - Sir Henry et leurs adjoints:

$M_{1}=$ Ducoudray; $M_{2}=$ Duthier et $M_{3}=$ Vaughan,

il est possible de coupler les opposants suivants:

$B_{1}-M_{1}=$ les deux journalistes ont la même fonction actantielle: changer de camp pour rétablir l'équilibre du système original ${ }^{20}$.

$\mathrm{B}_{2}-\mathrm{M}_{2}=$ de retour d'Ottawa, Marguerite est empoisonnée à la place de Lamirande par un complice de Duthier, lui-même tué à la place dé Lamirande dans le train d'Ottawa.

$B_{3}-M_{3}=c$ 'est en ressuscitant aux yeux de Vaughan que Marie provoque la conversion de ce dernier (et, avec son changement de camp, la victoire des séparatistes). 
On obtient donc le schéma actantiel de Pour la Patrie.

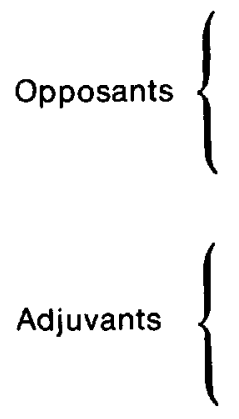

Héros: $M-M^{\prime}$

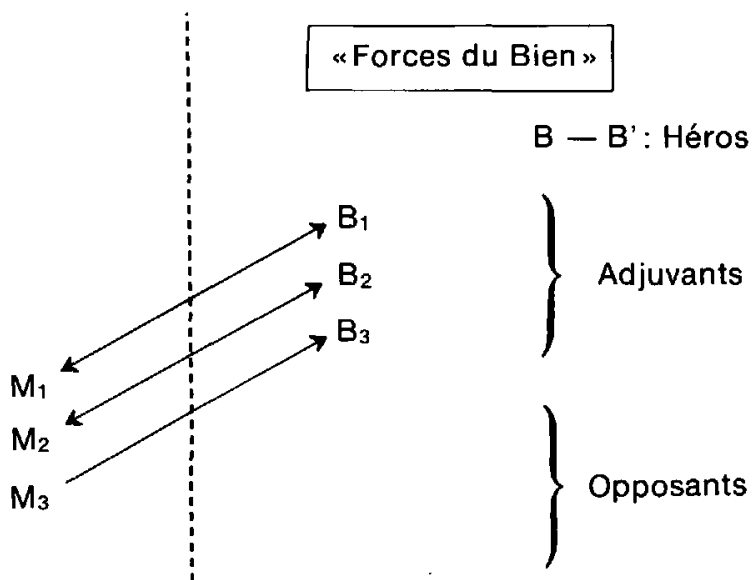

«Forces du Mal»

Les forces du premier couple d'opposants s'annulant (chacun changeant de camp), le second couple est amené à disparaître à son tour (décès de Marguerite et de Duthier). Seul Vaughan, du troisième couple, reste en liste (ou en lice) pour faire pencher la balance du bon côté. Mais Marie $\left(B_{3}\right)$ retourne au Ciel:

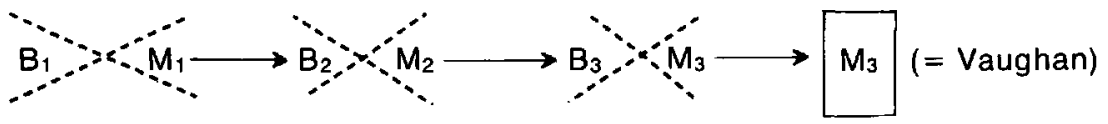

Avec la victoire des séparatistes (= la réalisation du projet initialement encodé), s'ensuit la disparition de trois des héros:

M - Montarval se suicide

M' - Sir Henry démissionne (et disparaît de la diégèse);

$B$ - Lamirande se retire dans un monastère français .

$\mathrm{Ne}$ restent plus du bon côté que B' (Leverdier) et l'ex-M $\mathrm{M}_{3}$ (Vaughan). $\mathrm{Ce}$ principe de "permutation-intégration" a donc entraîné la disparition en chaîne de tous les couples (adjuvants, opposants et adjuvants-opposants), pour stabiliser le système narratif avec un dernier couple B' - B'" = Leverdier-Vaughan (permutation de ce dernier, intégré aux «forces du Bien»). Remarquons la rigueur de cette dynamique allant jusqu'à l'élimination du personnage principal et - dans la mesure où Tardivel assume pleinement son idéologie - de l'auteur ou, tout du moins, du locuteur principal. C'est tout le problème de l'énonciation du texte, sur lequel je terminerai cette étude. 
“Et les deux amis partirent pour Ottawa, fermement convaincus que Lamirande les y rejoindrait bientôt. Mais ils ne le virent plus jamais, ni à Ottawa, ni ailleurs. »

Suit la lettre d'adieu du héros dont la signature «Joseph Lamirande» ponctue ce dernier chapitre, rappelant curieusement la ponctuation de l'Avant-propos signé: «J.-P. Tardivel». Il est significatif, par ailleurs, que le nom de Lamirande ponctue le dernier chapitre et celui du Frère Jean l'Épilogue: tout se passe comme si Lamirande avait bel et bien cessé d'exister (il a laissé son testament politique au dernier chapitre) après la dernière mention de son nom (référant à un personnage encore vivant). L'Épilogue qui prolonge pourtant la diégèse avec le traditionnel bilan des romans d'aventure (que sont devenus $X, Y, Z \ldots$ ?), ne mentionne jamais Lamirande qu'au passé. Seul le mystérieux locuteur (un moine?) qui assume la clôture du texte, se doute de l'identité du Frère Jean: “J'étais entièrement persuadé que nous descendions la montagne en compagnie du héros de la Nouvelle-France; et j'étais fortement tenté, je l'avoue, de faire part de ma conviction à mes compagnons de route. Mais je résistai à la tentation [...] Ne serait-ce pas une sorte de profanation? J'eus la force de retenir ma langue ${ }^{21}$, »

C'eût été effectivement une profanation, mais elle n'eût pas consisté à «arracher à ce bon frère le secret que Dieu lui a permis de garder si longtemps". C'eût été une faute autrement plus grave contre tout le système d'encodage du roman ${ }^{22}$. En refusant d'en dire trop long, le discours respecte la disparition du héros au niveau diégétique: Lamirande n'existe plus pour ses compagnons, même plus, dirons-nous, pour son locuteur premier (celui des chapitres 1 à 33). En effet, le corps de l'Épilogue est le fait d'un second locuteur. Une lettre est adressée à un journal français par un nouveau locuteur, témoin ayant recueuilli les propos de chartreux et reconstitué à partir d'une rencontre avec le Frère Jean, l'histoire de ce dernier. Cette lettre, le locuteur premier ne fait que l'enchâsser dans son récit, en se retirant discrètement du texte (il s'agit, pour lui comme pour Lamirande d'une retraite, le roman lui-même figurant son testament politique): sont encore de lui l'inter-titre "Épilogue», la citation latine propre à chaque chapitre ${ }^{23}$ et la phrase liminaire de l'Épilogue: «Dans son numéro du 15 février 1977, la Croix, de Grenoble, France, publia la communication suivante: [...]»

Dans cette lettre non signée, peu ou pas de ces fleurs de rhétorique auxquelles nous avait habitué le style du locuteur premier. Des faits sont communiqués. Le seul passage rappelant l'écriture première (récit de la mort du Frère Jean avec apparition de sa fille) se trouve assumé par d'autres moines (narration au troisième degré!), le locuteur second s'éclipsant à son tour, suivant en cela le principe de substitution mis à jour tout au long du texte: "Nous savions tout de suite, m'ont raconté les moines [...] 
Puis - je laisse la parole aux peres - [...] " Avec cette ultime passation des pouvoirs, le principe de permutation aura fonctionné jusqu'au bout. Entendons jusqu'au dernier paragraphe de la dernière page, id est jusqu'à l'exclusion de tous les permutants: substitution de Lamirande par Leverdier au Québec et par le Frère Jean en France, avec éclipses successives des locuteurs entre eux.

Le texte se mord la plume en une étonnante clôture ${ }^{24}$, ne laissant au lecteur confondu qu'un étrange sourire dont on se demande - malgré la confusion à éviter entre actant, locuteur et auteur, et en dépit du sérieux de ce dernier - s'il n'est pas de moquerie: "comme un souffle lumineux son âme quitta son corps [...] il n'y avait de surnaturel dans la cellule que le sourire qui illuminait les traits du frère Jean".

John Hare rapporte qu'un mois après la mort de Tardivel, Pour la Patrie demeurait pour l'Artisan "un roman très étrange où l'on ne sait si l'auteur est sérieux ou s'il veut mystifier ${ }^{25}$ ».

1. Il s'agit, synchroniquement, des normes admises au moment de la production du texte, en l'occurence, à la fin du XIX ${ }^{e}$ siècle littéraire québécois (1895).

2. Paul Wyczynski, Panorama du roman canadien-francais, dans le Roman canadien-français, Montréal, Fides, tome III des "Archives des lettres canadiennes ", p. 13 à 16.

3. Sur les conditions socio-culturelles d'émergence du texte, voir la Présentation de John Hare, p. 9 à 45 de l'édition HMH, 1976.

4. Philippe Lejeune, le Pacte autobiographique, Paris, Seuil, 1975, p. 8.

5. Pour la Patrie, p. 50 et 51 (nous désignerons désormais ce texte par $P L P$ ).

6. Tardivel signale lui-même les parangons de son "nouveau roman ": le Roman d'un Jésuite (de Gabriel de Beugny d'Hagerue, Paris, Palmé, 1887) et JeanChristophe (de Paul Deschamps, Langres, Maitries et Courtot, 1893). Je souligne "contre-partie" en rappel du principe de permutation dégagé plus haut.

7. PLP, p. $50-51$.

8. Présentation de PLP par J. Hare, p. 24, qui signale pour les manifestations majeures de cette idéologie: $M^{9 r}$ Laflèche, Quelques considérations sur les rapports de la société civile avec la religion et la famille (1866) et Philippe Masson, le Canada français et la Providence (Québec, 1875).

9. J. Hare, op. cit. p. 24. La citation interne est de R. Ruyer, l'Utopie des utopies, Paris, PUF, 1950, p. 206.

10. Mgr Justin Fèvre, Vie et travaux de J.-P. Tardivel, fondateur du journal la Vérité à Québec, Paris, Savaète, 1906. (Cité par J. Hare)

11. N'oublions pas que le roman est perçu comme un attribut des «forces du Mal $m$ !

12. Avec "notre premier roman canadien d'origine française: I'Influence d'un livre" (David Hayne, les Origines du roman canadien-français, dans les "Archives " citées à la note 3 (p. 37 à 72 ).

13. Dictionnaire encyclopédique des sciences du langage, Paris, Seuil, 1972, p. 196: "Et $X$ et non- $X$ serait la transgression du genre, ni $X$ ni non- $X$, du type. $)$

14. Op. cit.

15. Tardivel cite expressément dans l'Avant-propos l'exemple du dictateur équatorien. 
16. Dans le premier cas, ct. le début du chapitre 4 et le chapitre 21, p. 176 ; dans l'autre, cf. le début du chapitre 12, avant l'empoisonnement de Marguerite.

17. T. Todorov, Introduction à la litterature fantastique, Paris, Seuil, 1970, p. 61-62.

18. Ibid., p. 61.

19. ... clôture édifiante du chapitre 26 !

20. On peut objecter qu'ancien catholique passé franc-macon, Ducoudray avait déjà rompu l'équilibre dans une temporalité pré-diégétique. Notre analyse portant sur la seule durée diegetique, il nous est permis de n'en pas tenir compte. Du reste, sa situation d'agent-double (et sa position encombrante dans l'économie du récit) sera réglée par son assassinat: c'est le seul transfuge qui mérite (textuellement) son sort.

21. PLP, p. 270 . (Je souligne la périphrase référant à Lamirande, ainsi que la formule de réticence, toute rhétorique: retenir sa langue.)

22. On se rappelle la problématique analogue du Survenant, de G. Guèvremont, dont la seule édition posthume propose la résolution de l'énigme (l'identité du héros), en rupture avec tout l'encodage du système romanesque.

23. Citation du dernier chapitre: “Cursum consummavi. J'ai achevé ma course, (II TIM IV, 7)"!

24. On ne peut s'empêcher d'admirer la perfection de cette structure qui comble (corrige) au dernier moment le seul "manque" du texte: la séquence ouverte au chapitre 30 (en repartant au Ciel, Marie avait annoncé à son père: "Nous irons ensemble au Paradis») est refermée dans l'Épilogue ( I'Enfant-Jésus m'a envoyé vous chercher. Venez!m).

25. Cité par Mgr Fèvre, op. cit., p. 215. 